\title{
Research on the Application Development of Intelligent Technology in Electrical Automatic Control Engineering
}

\author{
Wenhui Xie \\ Guangdong Technician College, Huizhou, Guangdong, 516100, China \\ xwhgdsgj@163.com
}

Keywords: Electrical Automatic Control, Intelligent Technology, Application Development

\begin{abstract}
With the development and progress of computer science and technology, intelligent technology has been widely used in real life, especially in the process of construction need to attach great importance to electrical installation construction, this is a key link, which will have a great impact on the convenience and practicality of the system. This paper is mainly about the research and analysis of intelligent technology application in electronic automation control engineering for reference and reference of relevant professionals.
\end{abstract}

\section{Introduction}

The control methods and methods adopted in the electrical automation control engineering are relatively traditional, but if the intelligent technology is adopted at present, it is often more reasonable and scientific than the traditional control, and it is also more convenient and quick. In the process of the current electrical automation control, it is necessary to further strengthen the research and application of the intelligent technology. Only in this way, can the electrical automatic control project play a good role in improving the quality and effect of electrical control.

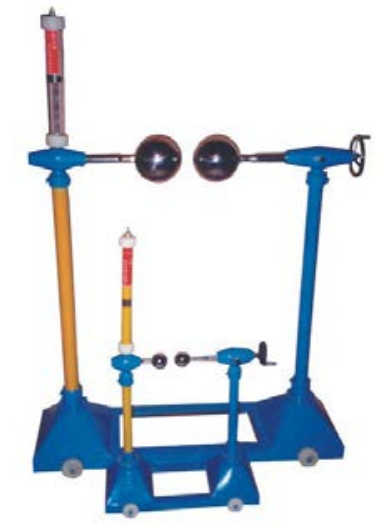

Figure 1 Electrical automation control

\section{Intelligent Technology}

Intelligent technology is a new modern technology that promotes the combination of various technologies and multi-disciplinary knowledge. In the process of people's life and work, it can play a good application effect by using intelligent technology, and at the same time, it is far beyond the scope of manpower. With the development of social economy and science and technology, intelligent technology has been developed rapidly, and has achieved great perfection and innovation, and has many advantages, which can play a good role in electrical engineering. First of all, intelligent technology does not need to build a control model. Generally speaking, the traditional electrical automatic control needs the model, at the same time, the controlled object and the control point are connected with each other, so the relatively precise parameters are often set, which can effectively improve the control effect and precision of the model. However, the use of intelligent technology in the current process of automatic electrical control can achieve precision control, but 
also can achieve automatic control of electrical equipment. Compared with the traditional electrical control, the wide application of intelligent technology can significantly improve the efficiency and quality of equipment control. Secondly, through the use of intelligent technology to achieve rapid control and adjustment of electrical systems, the use of intelligent technology in the current electrical automatic control project can promote the electrical equipment operation data to achieve rapid and effective analysis and statistics, but also through the relevant reference data to ensure the electrical system to achieve healthy and stable operation. In addition, the use of intelligent technology in the current electrical control project can also achieve all-round collection of electrical equipment information, which is conducive to intelligent analysis of data parameters and information, while combining with the actual situation to make scientific and effective adjustments to ensure that the electrical automatic control project to achieve efficient, reliable and stable operation. In addition, the application of intelligent technology in the current electrical automatic control project, as long as the input of data information can be through the use of unified automatic control standards for information and data scientific and effective evaluation, which fully shows that intelligent technology in the process of processing different data itself has the characteristics of consistency, the staff can combine the analysis results for the electrical control project to achieve targeted control, and then achieve idealized control effect[1].

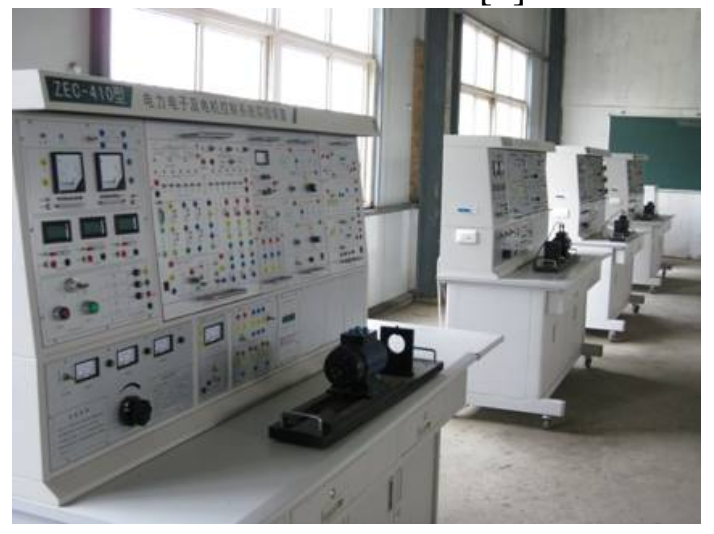

Figure 2 Electrical control engineering

\section{Related Contents of Intelligent Technology in Electrical Automatic Control Engineering}

\subsection{Intelligent Introduction of Electrical Automatic Control Engineering}

With the development of social economy and science and technology at present, in order to make the current electric automation engineering conform to the trend of the times, it is necessary to combine the electric automation control and intelligent technology at present, so as to construct the intelligent network with the characteristics of automation, digitization and informationization. In the current electronic control engineering, the application of intelligent technology can ensure the normal and stable operation of the equipment. In the process of the actual construction of electrical engineering, it is necessary to realize effective management and control in many aspects of the application, especially in the dynamic aspect of the system, so as to effectively prevent many bad problems and contents in the process of electrical engineering construction.[3].

\subsection{Intelligent Advantage of Electrical Automatic Control Engineering}

Compared with the previous electronic control equipment, the intelligent technology itself has the characteristics of energy saving, environmental protection and intelligence. For the traditional electrical control equipment machines, through the use of intelligent equipment can better quickly detect the bad situation in the grid equipment, but also can quickly and effectively feedback the bad situation to the superior management. The use of modern intelligent technology can effectively ensure that the relevant staff to achieve good control of electrical control equipment, in addition to the use of automatic intelligent technology, but also to promote the use of equipment service life can be greatly improved, can save costs, in the actual work process, the relevant staff can 
effectively use intelligent technology to reduce the workload, more environmentally friendly than traditional equipment, conducive to the combination of environmental protection and intelligence, which is a major development trend of automation control engineering.

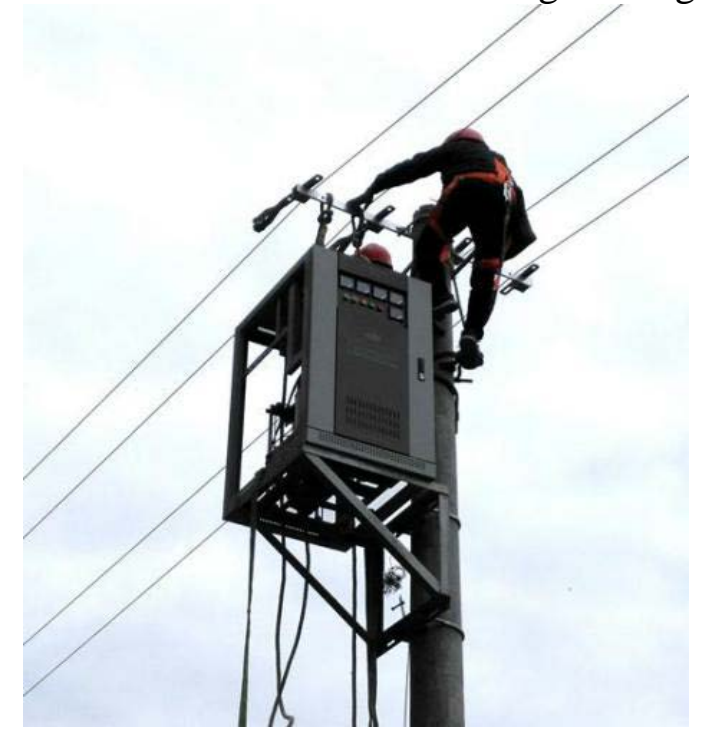

Figure 3 Electrical control operation flow

\section{The Practical Content of Intelligent Technology Application in Electrical Automatic Control Engineering}

\subsection{The Application of Intelligent Technology in the Design of Electrical Optimization}

Compared with the traditional electronic control system, the design of the traditional electrical control system requires that the relevant staff have a wealth of relevant knowledge and experience, but even if the personnel with higher quality, it is still relatively difficult to design a relatively excellent system, in the current electrical automation control project, we should strengthen the wide use of intelligent technology, in the process of realizing the design of electrical automation system, staff should be able to use computer technology to carry out reasonable scientific design for the fine part of the system, so as to reduce the time of system design obviously. In addition, the CPU of the central processing unit is the processing center of the intelligent electrical system, and the data can be input into the circuit by using the central processing unit, and the data collection can be realized quickly, and the effect and quality of the work can be significantly improved by using the new system.

\subsection{Application of Intelligent Technology in Electrical Fault Diagnosis}

The electrical control system should invest a lot of financial resources and manpower, it needs a lot of cost and money, in the process also need the relevant staff to realize the precision calculation of electrical control, and all of this needs to rely on the staff to carry out the work, the results and data in it may cause certain errors, but the use of intelligent technology in electrical automation control engineering is more reliable and reliable than artificial, which is beneficial to improve the accuracy and realize the intelligent control of electrical automation engineering. Compared with the traditional automation control, it is more intelligent and efficient, which is beneficial to save cost and cost.

\subsection{The Application of Intelligent Technology in Electrical Control}

In the current process of fault diagnosis, it is necessary to strengthen the use of intelligent technology widely. At present, it is a relatively difficult link for the electrical automation fault diagnosis itself. It is necessary to locate the fault accurately, and to strengthen the checking work further. However, the use of intelligent technology in the current process of electrical automatic control, the use of new equipment and materials in the process of electrical automation control, but 
also to strengthen the application of computer technology, conducive to the equipment to achieve reliable detection and analysis, but also rapid collection of equipment real-time data and real-time state, can effectively determine the failure of the system, effectively meet the relevant staff to implement repair and check requirements, which is of great value and significance for ensuring the smooth operation of electrical automation control project.

\subsection{Application of Intelligent Technology in Risk Prediction}

In the process of motor fault diagnosis and analysis, intelligent technology can play an important role, through the use of diversified integrated technology can promote the ability to deal with, aggregate, collect and analyze greatly, but also can significantly improve the defense effect and defense ability of intelligent system, in addition, the risk prediction will be greatly improved and improved, once the input of relatively trustworthy means of treatment and analysis, can effectively ensure the smooth development and implementation of equipment maintenance, improve the economic benefits of modern enterprises, so as to achieve enterprise health, stability and sustainable development.[4].

\section{Conclusion}

To sum up, in the process of actual production, it is necessary to further strengthen the wide application of electrical automatic control project, which is directly related to the healthy and stable development of our national economy. At present, intelligent technology is a major development trend of the times, through the use of intelligent technology can effectively reduce human labor, but also avoid human error, so it is necessary to further strengthen the use of intelligent electrical automatic control engineering intelligent technology, through the use of intelligent technology in risk prediction, electrical fault diagnosis, electrical control and motor design process can effectively save manpower, material and financial resources.

\section{References}

[1] Li Chao. Application analysis of intelligent technology in automatic control of electrical engineering. Electronic World, no. 18, pp. 66-67, 2019.

[2] To the army. Analysis of the application of intelligent technology in the automatic control system of electrical engineering. Electrical Technology and Economy, no. 01, pp. 18-20, 2019.

[3] Li Guanbin. Discuss the application of intelligent technology in the automatic control system of electrical engineering. Technological Innovation Bulletin, vol. 15, no. 34, pp. 10-11, 2018.

[4] Yu, Chuangyu. Analysis of the application of intelligent technology in the automatic control system of electrical engineering. Private Technology, no. 11, pp. 10-11, 2018. 